\title{
A Fanconi-Bickel syndrome patient with a novel mutation and accompanying situs inversus totalis
}

\author{
Tuğba Taştemel-Öztürk ${ }^{1}$, Berrak Bilginer-Gürbüz ${ }^{2}$, Özlem Tekşam ${ }^{1}$, Serap Sivri² \\ Department of ${ }^{1}$ Pediatrics, ${ }^{2}$ Division of Pediatric Metabolism, Hacettepe University Faculty of Medicine, Ankara, Turkey. \\ E-mail:t_tastemel@hotmail.com \\ Received: 19th February 2017, Revised: 27th March 2017, Accepted: 23th April 2017
}

\begin{abstract}
SUMMARY: Taştemel-Öztürk T, Bilginer-Gürbüz B, Tekşam Ö, Sivri S. A Fanconi-Bickel syndrome patient with a novel mutation and accompanying situs inversus totalis. Turk J Pediatr 2017; 59: 693-695.

Fanconi-Bickel syndrome is a rare autosomal recessive disorder of carbohydrate metabolism, caused by mutations in the SLC2A2 gene, that codes for the glucose transporter protein 2 (GLUT2). The disease is characterized by proximal renal tubular dysfunction, impaired glucose and galactose utilization, and accumulation of glycogen in the liver and kidney. Signs and symptoms of Fanconi-Bickel syndrome begin in infancy and include failure to thrive, hepatomegaly, hypophosphatemic rickets, and short stature. Here in we report a Turkish Fanconi-Bickel syndrome case who also has situs inversus totalis and a novel mutation that has not been described before.
\end{abstract}

Key words: Fanconi-Bickel syndrome, glycogen storage disease, situs inversus totalis, novel mutation.

Fanconi-Bickel syndrome (FBS) or glycogen storage disease type XI (FBS; MIM 227810), a rare autosomal recessive disorder of carbohydrate metabolism, was originally described in 1949 by Fanconi and Bickel. ${ }^{1}$ In 1997 it was demonstrated that this syndrome is caused by mutations in the SLC2A2 gene, that codes for the glucose transporter protein 2 (GLUT2), which transports glucose in and out of hepatocytes, pancreatic $\beta$ cells, and the basolateral membranes of intestinal and renal epithelial cells. ${ }^{2}$ The structural gene encoding GLUT2 has been cloned and mapped to chromosome $3 \mathrm{q} 26.1-26.3 .^{3}$

The disease is characterized by proximal renal tubular dysfunction, impaired glucose and galactose utilization, and accumulation of glycogen in liver and kidney. Signs and symptoms of FBS begin in infancy and include failure to thrive, hepatomegaly, hypophosphatemic rickets, and short stature. Characteristic laboratory findings of the disease are glucosuria, phosphaturia, generalized aminoaciduria, bicarbonate wasting and hypophosphatemia due to proximal renal tubular dysfunction and increased serum alkaline phosphatase levels and radiologic findings of rickets. Mild fasting hypoglycemia and hyperlipidemia may also be present. ${ }^{4}$

Herein we report a Turkish Fanconi-Bickel syndrome case who has also situs inversus totalis and a novel mutation that has not been described before.

\section{Case Report}

A three-year-old male child, first child of second degree consanguineous parents, was admitted to our hospital at the age of three years, presented with abdominal distension, failure to thrive, motor and mental developmental delay and polyuria, polydipsia. There was no history of antenatal problems during the mother's pregnancy. He was delivered at full term with a birth weight of $3.25 \mathrm{~kg}$. Protuberant abdomen was noticed first at twelve months of age and situs inversus totalis and hepatomegaly were detected at this time.

On physical examination, length $79 \mathrm{~cm}(<3 \mathrm{rd}$ centile for age), weight $12.4 \mathrm{~kg}$ (3-10th centile for age), head circumference $49 \mathrm{~cm}$ (50th centile for age) were noted. On abdominal examination, the liver was $4 \mathrm{~cm}$ below the left costal margin while the spleen was palpable 2 
$\mathrm{cm}$ below the right costal margin. The heart sounds were on the right side of thorax. He was able to walk with support. The rest of the systemic examination were normal.

Laboratory assessment revealed a reduced fasting blood glucose $(38 \mathrm{mg} / \mathrm{dl})$, normal serum calcium, reduced serum phosphorus (2.6 mg/dl, normal: 3.1-6), elevated serum alkaline phosphatase (473 U/L, normal: 104$345)$, and elevated triglyceride $(262 \mathrm{mg} / \mathrm{dl})$ levels. Liver and kidney function tests were all normal. Arterial blood gas analysis was normal. Glucosuria and proteinuria were found in the urine stick test; generalized aminoaciduria was detected in the urine amino acid chromatography. Urine reducing substance was found positive and the chromatography revealed that it was glucose. Delayed bone age, rachitic changes at wrist joints were detected on the radiographic studies. Enlarged liver and situs inversus totalis were confirmed by ultrasonography and echocardiography. On the basis of these findings, FBS was presumed and was finally confirmed by molecular analysis of the GLUT2 (SLC2A2) gene. Direct sequencing of genomic DNA revealed a G-to-A substitution at position +5 of the splicing acceptor site in intron 2 of the GLUT2 gene in a homozygous pattern (IVS2+5G $>$ A [c. $108+5 \mathrm{G}>\mathrm{A}]$ ).

Oral potassium citrate (for potassium replacement), phosphorus supplementation and calcitriol treatment was started, and the patient has also been advised to have a modified cornstarch product. Carnitine was added to the treatment because carnitine level was close to the lower border. An informed consent was obtained from the parents of our patient about this report.

\section{Discussion}

To date, more than one hundred Fanconi-Bickel syndrome patients and 59 or more mutations which are responsible for the syndrome have been reported.5,6 Our patient has a novel mutation that was not described previously. Although an expression study of mRNA of this patient has not been performed yet, with our patient clinical manifestation we thought this mutation result in a splicing error. There are also patients reported who have clinical manifestations of Fanconi-Bickel Syndrome and no mutation are detected in SLC2A2 gene. $5,7,8$
The presence of a few SLC2A2 gene mutations that causes milder clinical course is also known. Grünert et al. ${ }^{9}$ described two siblings, first of them had failure to thrive at 9 months and glucosuria, mild tubular proteinuria with elevated transaminases. He had no other clinical manifestations of Fanconi-Bickel syndrome, such as rickets, hepatomegaly, renomegaly, hypoglycemia and postprandial hyperglycemia. Molecular analysis of this patient resulted as GLUT2 compound heterozygosity in SCL2A2 and owing to this patient his sister was investigated with in first month of life and found same compound heterozygote mutation similarly to her brother. This disease has a broad spectrum of severity, Fridman et al. ${ }^{6}$ determined that a group of 8 patients have the same mutation and have different phenotypic expression and suggested that the broad spectrum of disease severity among these patients might be explained by modifier genes, as suggested for other monogenic diseases or unknown epigenetic factors.

Clinical presentation of Fanconi-Bickel syndrome has been thought to be homogenous but different presentations and findings were reported in the literature, such as bilateral nuclear cataracts during neonatal period and transient neonatal diabetes. ${ }^{10,11}$ Our patient had a classical presentation with fasting hypoglycemia, hepatomegaly, proximal tubular disfunction, rickets and severe growth retardation; but had accompanying situs inversus totalis, interestingly. To our knowledge, the concomitance of these two conditions has not been reported before. The question is if this concomitance is a coincidence or an association. Situs inversus occurs in 1 out of $6000-8000$ newborns. The data about the genetics of isolated situs inversus is limited. Many of the genes that are known cause syndromic situs inversus; such as ciliopathies, including primary ciliary dyskinesia, nephronophthisis, polycystic kidney disease 2 and Bardet-Biedl syndrome. ${ }^{12}$ One of the mutant genes that causes primary ciliary dyskinesia and situs inversus, CCDC39 gene is mapped to chromosome $3 \mathrm{q} 26.33 .^{13}$ The mutant gene that causes Fanconi-Bickel syndrome is also mapped to chromosome 3q26.1-q26.3, and we considered if our patient's mutation might have a modifier gene impact on CCDC39 gene. 
There is no specific therapy for FBS. Symptomatic replacement of fluid, electrolytes, phosphate, sodium bicarbonate and vitamin D and restriction of galactose is recommended. Patients should avoid fasting and should be given mild frequent feedings and uncooked starch at bedtime to prevent hypoglycemia; this also helps in regression of hepatomegaly. We started treatment with oral potassium citrate, phosphorus supplementation and calcitriol and advised to have frequent complex carbohydrate feedings. We also started carnitine supplementation in order to treat carnitine deficiency secondary to tubulopathy.

\section{REFERENCES}

1. Fanconi G, Bickel H. Chronic aminoaciduria (amino acid diabetes or nephrotic-glucosuric dwarfism) in glycogen storage and cysteine disease. Helv Paediatr Acta 1949; 4: 359-396.

2. Santer R, Schneppenheim R, Dombrowski A, Götze $\mathrm{H}$, Steinmann B, Schaub J. Mutations in GLUT2, the gene for the liver-type glucose transporter, in patients with Fanconi-Bickel syndrome. Nat Genet 1997; 17: 324-326.

3. Fukumoto H, Seino S, Imura $\mathrm{H}$, et al. Sequence, tissue distribution, and chromosomal localization of mRNA encoding a human glucose transporter-like protein. Proc Nat Acad Sci U S A 1988; 85: 5434-5438.

4. Santer R, Schneppenheim R, Suter D, Schaub J, Steinmann B. Fanconi-Bickel syndrome--the original patient and his natural history, historical steps leading to the primary defect, and a review of the literature. Eur J Pediatr 1998; 157: 783-797.
5. Santer R, Groth S, Kinner M, et al. The mutation spectrum of the facilitative glucose transporter gene SLC2A2 (GLUT2) in patients with Fanconi-Bickel syndrome. Hum Genet 2002; 110: 21-29.

6. Fridman E, Zeharia A, Markus-Eidlitz T, Cohen YH. Phenotypic Variability in Patients with Fanconi-Bickel Syndrome with Identical Mutations. JIMD Rep 2015; 15: 95-104.

7. Akagi M, Inui K, Nakajima S, et al. Mutation analysis of two Japanese patient with Fanconi-Bickel syndrome. J Hum Genet 2000; 45: 60-62.

8. Ozer EA, Aksu N, Uclar E, et al. No mutation in the SLC2A2 (GLUT2) gene in a Turkish infant with Fanconi- Bickel syndrome. Pediatr Nephrol 2003; 18: 397-398.

9. Grünert SC, Schwab KO, Pohl M, Sass JO, Santer R. Fanconi-Bickel syndrome: GLUT2 mutations associated with a mild phenotype. Mol Genet Metab 2012; 105 : 433-437.

10. Furlan F, Santer R, Vismara E, et al. Bilateral nuclear cataracts as the first neonatal sign of Fanconi-Bickel syndrome. J Inherit Metab Dis 2006; 29: 685.

11. Sansbury FH, Flanagan SE, Houghton JAL, et al. SLC2A2 mutations can cause neonatal diabetes, suggesting GLUT2 may have a role in human insulin secretion. Diabetologia 2012; 55: 2381-2385.

12. Deng H, Xia H, Deng S. Genetic basis of human left-right asymmetry disorders. Expert Rev Mol Med 2015; 16: e19.

13. Merveille AC, Davis EE, Becker-Heck A, et al. CCDC39 is required for assembly of inner dynein arms and the dynein regulatory complex and for normal ciliary motility in humans and dogs. Nature Genetics 2011; 43: $72-78$. 